\title{
The Effect of Behavior Conformity on Prisoner's Dilemma Game
}

\author{
Zidong Chai, Yanbo Zhang*, Xinyu Zhang and Xiaobo Liu \\ School of Physics and Electronics, Henan University, Kaifeng, China \\ ${ }^{*}$ Corresponding author
}

\begin{abstract}
Explaining the evolution of cooperative behavior is one of the most important and interesting problems in a myriad of disciplines. Game players on complex networks usually change their strategies by copying special rules. In this research, we combine the prisoner's dilemma game player's behavior conformity with the Fermi process. The game player changes the strategy by choosing a Fermi process or behavior consistency by a certain probability. And we have researched the evolutionary process of game players' cooperative behavior on a regular network of different sizes. The analysis of the data shows that the demand for behavior conformity is conducive to the emergence of the cooperative behavior of the game groups in prisoner's dilemma. Cooperative phenomenon is of great significance for intelligent machine collaboration.
\end{abstract}

Keywords—prisoner's dilemma; behavior conformity; network.

\section{INTRODUCTION}

In social group, cooperation is one of the important factors of group survival and reproduction. Human sometimes give support to help others without any feedback[1]. These actions will consume personal assets some. Mutual cooperation among members of the group, human and social species have achieve success of evolutionary success. Cooperation makes our lives better[2]. However, the formation of scope cooperation in the group is still difficult, and the prisoner's dilemma reveals the relationship of cooperation and betrayal among individuals. In the basic model, each player must simultaneously choose one strategy between cooperation $(\boldsymbol{C})$ and defection $(\boldsymbol{D})$. If both of them choose cooperation (or defection), they will get the reward $\mathrm{R}$ (or defection $\mathrm{P}$ ). However, if one cooperator meets one defector, the former gets sucker's payoff $\boldsymbol{S}$, while the later gets Temptation $\boldsymbol{T}$. If the payoff ranking satisfy $\boldsymbol{T}>\boldsymbol{R}>\boldsymbol{P}>\boldsymbol{S}$ and $2 \boldsymbol{R}>\boldsymbol{T}+\boldsymbol{S}$, it will be the prisoner's dilemma game; It is obvious that, mutual defection and cooperation-defection pair optimizes the individuals' payoff in prisoner's dilemma, although mutual cooperation could yield a higher collective benefit. Thus, the survival of cooperation seems to be still difficult[3].

When choosing a kind of behavior, the actual individual not only considers the objective income that the behavior brings to himself, but also collects the relevant information of the people around them[4]. The communication of this information is one of the most important ways to achieve competition or cooperation. If the individuals around are the same as their own behavior, the individual would gets a greater sense of satisfaction. Otherwise, the experience feels that he is incompatible with the environment and produces subjective uncomfortable. This psychology makes the individual prefer to choose the strategy of most people around[5]. The ways of the individual's own behavior strategies are complex and diverse, conformance, namely, behavior conformity is one of the most common kind ways[6]. Based on this, the main research of this paper: In the regular network model, The effect of behavior consistency and replication dynamics (Fermi rules) on the evolution of group behavior. Through experimental simulation, The evolution of group cooperative behavior on a homogeneous small world network based on grid networks is studied.

\section{STRUCTURE}

We consider the evolutionary prisoner's dilemma game in this study. The payoff matrices for both games are shown in the following Table.1:

\begin{tabular}{ccc} 
TABLE I. & GAIN MATRIX \\
\hline $\begin{array}{c}\text { strateg } \\
\text { ies }\end{array}$ & $\mathrm{C}$ & $\mathrm{D}$ \\
\hline $\mathrm{C}$ & $\mathrm{R}$ & $\mathrm{S}$ \\
$\mathrm{D}$ & $\mathrm{T}$ & $\mathrm{P}$ \\
\hline
\end{tabular}

Table.1: The evolutionary prisoner's dilemma game is assigned as follows: the temptation $\boldsymbol{T}=2$ if one player defects while his opponent cooperates, reward $\boldsymbol{R}=1$ if both cooperate, and both the punishment $\boldsymbol{P}=0$ for mutual defection as well as the sucker's payoff $\boldsymbol{S}=-2$.

Players are located in lattices with $\boldsymbol{L} * \boldsymbol{L}$ units. Each player adjusts or maintains strategy by comparing with the gain of neighborhood after each round of game[7]. Self-interest maximization is the rational player's pursuit in game, this is determined by player's need for development. Otherwise some rational players may occasionally make a certain degree of error when they change strategy, through statistics and analysis of the vast data, at present, most researchers use Fermi rules to express irrational imitation of players[8]. The game player $\boldsymbol{i}$ selects a neighbor $\boldsymbol{j}$ randomly from four neighbors, then the probability of the player $\boldsymbol{i}$ takes the strategy of neighbor $\boldsymbol{j}$ in the next game is:

$$
P_{(i \leftarrow j)}=\frac{1}{1+e^{\left[\left(u_{i}-u_{j}\right) / k\right]}}
$$


$\boldsymbol{u}_{\boldsymbol{i}}$ and $\boldsymbol{u}_{\boldsymbol{j}}$ represent gain of player $\boldsymbol{i}$ and $\boldsymbol{j}$ respectively. $\boldsymbol{K}$ quantifies the uncertainty by strategy adoption, which indicates that the player chooses irrationally. The probability of irrational selection increases with the increasing of value $\boldsymbol{K}[8,9]$. In the $\boldsymbol{K} \rightarrow 0$ limit, player $\boldsymbol{i}$ copies the strategy of player $\boldsymbol{j}$ only when $\boldsymbol{u}_{\boldsymbol{j}}>\boldsymbol{u}_{\boldsymbol{i}}$. Conversely, in the $\boldsymbol{K} \rightarrow \infty$ limit, payoffs cease to matter and strategies change as per flip of a coin[10].

In the evolutionary mechanism proposed in this paper: Game players in each round of game, there is a probability of p0 for selecting behavior conformity strategy, and a probability of ( $1-\boldsymbol{p} \mathbf{0})$ for selecting Fermi rules to replace the probability of choosing their own strategies. The formula of behavior consistency are described as follows:

$$
\frac{(k c-k d)}{k i^{2}}>=c m p
$$

In the above formula, $\mathbf{c m p}$ is the degree of demand for individual behavior conformity. The number of individual neighbors choosing $\boldsymbol{C}$ strategy is $\boldsymbol{k} \boldsymbol{c}$, and $\boldsymbol{k} \boldsymbol{d}$ is the number of $\boldsymbol{D}$ chooser number, $\boldsymbol{k i}$ is the number of neighbors besides individual. When players use behavioral conformity to change strategies, the formula (2) is established, and the game player will choose the $\boldsymbol{C}$ strategy. Otherwise, game player will choose $\boldsymbol{D}$ strategy. When $\boldsymbol{L}=10$ (number of player $=\boldsymbol{L} * \boldsymbol{L}$ ), and the density of the cooperator at initialization is $0.5, \boldsymbol{R}=1, \boldsymbol{S}=-2$, $\boldsymbol{T}=2, \boldsymbol{P}=0, \boldsymbol{c m p}=0.1, \boldsymbol{p} \mathbf{0}=0$. The statistical results of the game players' strategy ratio in each game are shown as below Figure 1:

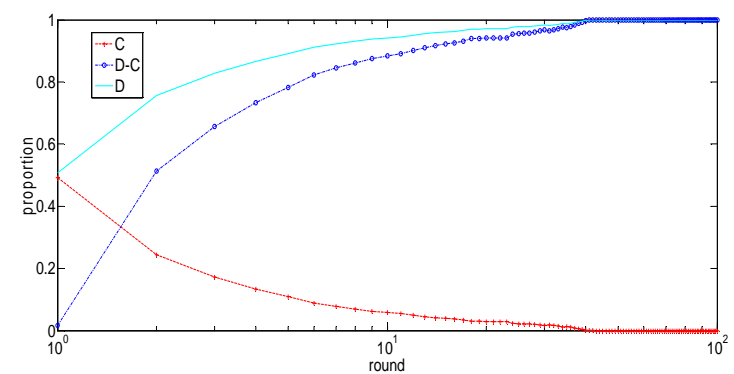

FIGURE I. THE GAME PLAYERS' STRATEGY RATIO IN EACH GAME

Figure 1. For everyone in our game selects their strategies obey the Fermi rules. When $\mathbf{p} \boldsymbol{0}=0$, in the range of $(0-80)$ rounds, the proportion of $\boldsymbol{D}$ (blue) selector is waved in 50 to $100 \%$, and reached the peak in round 80 , the selectors of $C$ (red) will disappeared in $(0-80)$ rounds. That means to pursue profit maximization inflexibly would evolve the betrayal of the whole because of the use in Fermi.

To analyze the useful of $\boldsymbol{p} \boldsymbol{0}$, we paid attention to the cooperative evolution in the network when $\boldsymbol{p} \mathbf{0}$ means different figure, $\left(N_{D}-N_{C}\right) / L^{2}$ means the selectors who choose betrayal subtracts the selectors of cooperation.

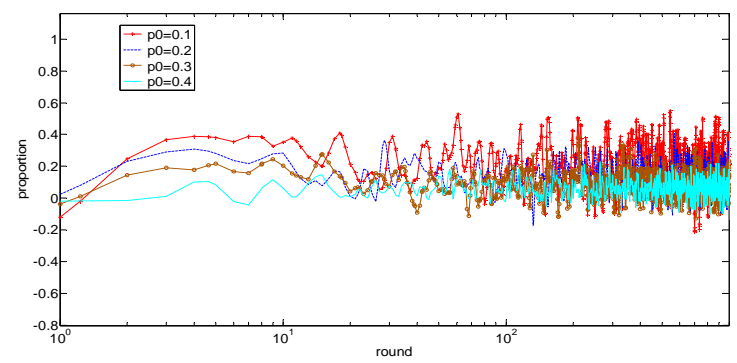

FIGURE II. CHANGING SITUATION OF $\left(\mathrm{N}_{\mathrm{D}}-\mathrm{N}_{\mathrm{C}}\right) / \mathrm{L}^{2}$ IN DIFFERENT P0

Figure 2. When $p 0=0.1($ red $)$, the average of $\left(N_{D}-N_{C}\right) / L^{2}$ is 0.2257 in $0<$ round $<1000$ and the variance is 0.0202 ; when round $>18$, the value of $\left(N_{D}-N_{C}\right) / L^{2}$ is between -0.24 (round $=701)$ and 0.58 (round $=544)$. When $p 0=0.2$ (blue), the average is 0.1356 , the variance is 0.011 and the $\left(N_{D}-\right.$ $\left.N_{C}\right) / L^{2}$ is between -0.2 (round $=132$ ) and 0.48 (round $\left.=390\right)$. When $0=0.3$ (brown), the value of $\left(N_{D}-N_{C}\right) / L^{2}$ has arrived at the top of 0.44 (round $=212$ ) and has reached the peak valley of -0.2 (round $=711$ ), at this time, the average is 0.0870 and the variance is 0.0088 . When $\mathbf{p 0}=0.4$ (cyan), the top value of $\left(N_{D}-N_{C}\right) / L^{2}$ is 0.34 (round $=264$ ) and the bottom is 0.16 , the average is 0.0594 and the variance is 0.0061 . Along with the increasing of $p 0$, the top value of $\left(N_{D}-N_{C}\right) / L^{2}$ has decreased from 0.58 to 0.34 and the bottom has increased from -0.24 to -0.16 , the average and the variance has let up. That all means when the range of cooperation has increased, the wave of $\left(N_{D}-N_{C}\right) / L^{2}$ has decreased.

When $\boldsymbol{L}=20$, the proportion of strategies distribution of game players simulation $\left(N_{D}-N_{C}\right) / L^{2}$ after fitting is shown as followed:

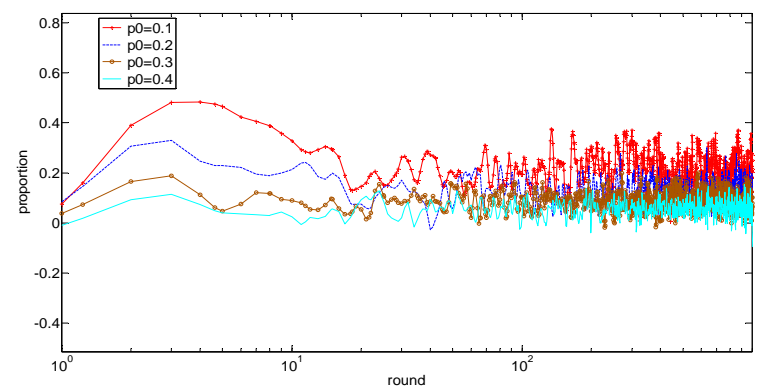

FIGURE III. CHANGING SITUATION OF (ND-NC)/L² IN DIFFERENT P0

Figure 3. When $p 0=0.1$ (red), the value of $\left(N_{D}-N_{C}\right) / L^{2}$ has arrived at the top of 0.485 (round $=5$ ) and then it decreased at the bottom of 0.281 (round $=12$ ); it raised again to 0.3 (round $=14$ ) and then it has fluctuate within the region of -0.01 (round $=443$ ) to 0.37 (round $=134$ ). The average of $\left(N_{D}-N_{C}\right) / L^{2}$ is 0.214 between 1000 rounds and the variance is 0.0055 . When $p 0=0.2$ (blue), the value of $\left(N_{D}-N_{C}\right) / L^{2}$ has arrived at the top of 0.3550 (round $=2$ ) and then it decreased at the bottom of -0.05 (round $=40$ ), the average is 0.1314 and the variance is 0.0031 . When $\mathbf{p 0}=0.3$ (brown), the value of $\left(N_{D}-N_{C}\right) / L^{2}$ has arrived at the top of 0.215 (round $\left.=668\right)$ and then it decreased at the bottom of -0.04 (round $=381$ ), the average is 0.0893 and the variance is 0.0021 . When $\mathbf{p 0}=0.4$ (cyan), the value of $\left(N_{D}-N_{C}\right) / L^{2}$ has arrived at the top of 
0.185 (round $=935$ ) and then it decreased at the bottom of 0.08 (round $=199$ ), the average is 0.0564 and the variance is 0.0017 .

Along with the increasing of $p 0$, the value of $\left(N_{D}-N_{C}\right) / L^{2}$ will decreased within limits, and the bottom of it is decreasing of the whole except when $\boldsymbol{p} \boldsymbol{0}=0.2$ and $\boldsymbol{p} \boldsymbol{0}=0.3$. We could see the wave range of $\left(N_{D}-N_{C}\right) / L^{2}$ in Figure 3, it is decreasing when $\boldsymbol{p} \boldsymbol{0}$ is increasing. That means the range of cooperation is also enlarged while $\boldsymbol{p} \boldsymbol{0}$ is increasing. When $\boldsymbol{L}=50$, the proportion of strategies distribution of game players simulation $\left(N_{D}-N_{C}\right) / L^{2}$ after fitting is shown as followed:

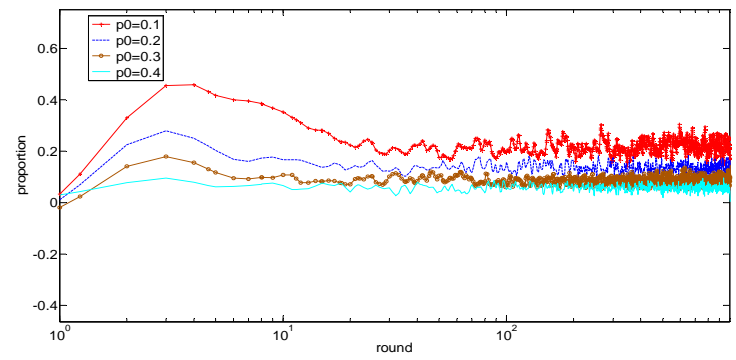

FIGURE IV. CHANGING SITUATION OF $\left(\mathrm{N}_{\mathrm{D}}-\mathrm{N}_{\mathrm{C}}\right) / \mathrm{L}^{2}$ IN DIFFERENT P0

From Figure 4, when $\mathbf{p} 0=0.1$ (red), the value of $\left(N_{D}-\right.$ $\left.N_{C}\right) / L^{2}$ has arrived at the top of 0.4632 (round $=4$ ), and then, within the increasing of the round, it has reached 0.21 (round $=21$ ). From then it has fluctuate within the region of 0.14 (round $=345$ ) to 0.30 (round $=265$ ), the average of it is 0.2213 between 1000 rounds and the variance is 0.0012 . When p0 $=0.2$ (blue), the value of $\left(N_{D}-N_{C}\right) / L^{2}$ has arrived at the top of 0.2688 (round $=3$ ), within the increasing of the round, it has reached 0.1647 (round $=25$ ). From then it has fluctuate within the region of 0.1031 (round $=35$ ) to 0.1714 (round $=$ 984), the average of it is 0.1351 and the variance is $5.5196 \times 10^{-}$ 4. When $\boldsymbol{p 0}=0.3$ (brown), the top of it is 0.1736 (round $=2$ ) and the average is 0.0876 , the variance is $3.6431 \times 10^{-4}$. When p0 $=0.4$, the top of it is 0.1144 (round $=757$ ) and the bottom of it is -0.0048 (round $=502$ ), the average is 0.0579 and the variance is $2.4668 \times 10^{-4}$. Within the increasing of $p 0$, the value of $\left(N_{D}-N_{C}\right) / L^{2}$ has decreased. The bottom is also decreasing except when $p 0=0.3$ (brown) and $p 0=0.4$ (cyan). That means the range of cooperation is also enlarged while $\boldsymbol{p} 0$ is increasing. With the increasing of $\boldsymbol{L}$, for example, when $\boldsymbol{p} \boldsymbol{0}=$ 0.1 :

TABLE II. THE DIFFERENT PARAMETER OF $\left(\mathrm{N}_{D}-\mathrm{N}_{\mathrm{C}}\right) / \mathrm{L}^{2} \mathrm{IN}$ DIFFERENT VALUE OF L

\begin{tabular}{ccccc}
\hline L & Max & Min & Mean & Var \\
\hline 10 & 0.58 & -0.24 & 0.2257 & 0.0202 \\
20 & 0.485 & -0.01 & 0.214 & 0.0055 \\
50 & 0.4632 & 0.0088 & 0.2213 & 0.0012
\end{tabular}

Table. 2 We can know from Table.2 that with the increasing of $L$, the top value of $\left(N_{D}-N_{C}\right) / L^{2}$ is decreasing from 0.58 to 0.4632 . It means the cooperation range has enlarged but the degree of it is lesser. The average of $\left(N_{D}\right.$ $\left.N_{C}\right) / L^{2}$ is about 0.21 and the variance of it is decreasing from 0.0202 to 0.0012 . From Figure 2, Figure 3 and Figure 4, we could see that the volatility of $\left(N_{D}-N_{C}\right) / L^{2}$ is reducing when the value of $\boldsymbol{L}$ is increasing, it means the stability of game players is strengthened.

\section{CONCLUSION}

In the state of the constant density of initial cooperator, the introduction of behavior conformity in the prisoner's dilemma game structure is helpful to the cooperative evolution of the group on the lattices. And to a certain extent resists the invasion of the betrayer. With the expansion of the network scale, the volatility of formula $\left(N_{D}-N_{C}\right) / L^{2}$ becomes smaller after the evolution of stability. It means that the strategy stability is strengthened, and it can promote group cooperation.

\section{ACKNOWLEDGMENTS}

This paper is supported by educational technology equipment and practice education in Henan (GZS029); key project of science and technology research of Henan Provincial Education Department (16A140004); teaching reform project of Henan University.

\section{REFERENCES}

[1] Dreber A, Rand D G, Rand D G, et al. Winners don't punish[J]. Nature, 2008, 452(7185):348-351.

[2] Perc M. Phase transitions in models of human cooperation[J]. Physics Letters A, 2016, 380(36):2803-2808.

[3] Ma Y, Lu J, Shi L. Diversity of neighborhoods promotes cooperation in evolutionary social dilemmas[J]. Physica A Statistical Mechanics \& Its Applications, 2017, 468:212-218.

[4] Coultas J C. When in Rome. An Evolutionary Perspective on Conformity[J]. Group Processes \& Intergroup Relations, 2004, 7(4):317331.

[5] Effersonabd C, Richerson P J, Mcelreath R, et al. Conformists and mavericks: the empirics of frequency-dependent cultural transmission [J]. Evolution \& Human Behavior, 2008, 29(1):56-64.

[6] Goyal, Sanjeev, Vega-Redondo, et al. Network Formation and Social Coordination[J]. Working Papers, 2003, 50(2):178-207.

[7] Liu C, Li R. Power-law distributed temporal heterogeneity of human activities promotes cooperation on complex networks[J]. Physica A Statistical Mechanics \& Its Applications, 2016, 457:93-100.

[8] Perc M, Gómez-Gardeñes J, Szolnoki A, et al. Evolutionary dynamics of group interactions on structured populations: a review[J]. Journal of the Royal Society Interface, 2013, 10(80):20120997.

[9] Szolnoki A, Perc M, Szabó G. Topology-independent impact of noise on cooperation in spatial public goods games[J]. Phys Rev E Stat Nonlin Soft Matter Phys, 2009, 80(2):056109.

[10] Kurzban R, Houser D. Individual differences in cooperation in a circular public goods game[J]. European Journal of Personality, 2001, 15(S1):S37-S52. 